\title{
Editorial
}

\section{Advances in Diagnostic and Experimental Breast Pathology}

In this issue of the Open Breast Cancer Journal we have assembled a group of manuscripts from experts in the field of diagnostic and experimental breast pathology to report on changes influencing clinical care and to provide new insights into the mechanistic underpinnings of mammary gland neoplastic progression. Harigopal and Chhieng, at Yale University, reviewed the changing face of breast cytology focusing, in part, on "the benefits and limitations of fine needle aspiration biopsies in diagnoses of breast lesions" as western societies increasingly abandon this technique for core biopsy interpretation. They weave into their historical tapestry the rise of molecular cytopathology and the continued use of cytology as part of intraoperative examination by pathologists.

Adams reporting from Emory University covers one of the newly rediscovered human breast premalignant conditions, flat epithelial atypia, an outgrowth of the attempt for better defining of columnar cell lesions through breast core biopsies. Perhaps most critically, the realization that all such lesions are not flat and that via morphologic, immunophenotypic, and molecular criteria, these lesions are better tied to breast neoplasia ending in invasive carcinoma is more fully explored.

Wei and Hammed take a different direction in breast pathology diagnoses by reporting on spindle cell lesions within this organ. They emphasize a broad range of lesions stretching from reactive conditions to the neoplastic and discuss entities such as fibromatosis and nodular fasciitis (traditionally thought of as soft tissue tumors) through pseudoangiomatous stromal hyperplasia, clearly a breast specific entity, and then turn to other, more exotic, entities including inflammatory myofibroblastic tumor and reactive spindle cell nodule ending with a highly malignant spindle cell carcinoma and angiosarcoma.

Transitioning from the diagnostic to experimental Yuan, Serra, and Frost report on the emerging realization that the loss of primary cilia appears to be critically important to many pathologic processes ranging from polycystic kidney disease and hepatofibrosis to mammary gland neoplastic progression. Work from their laboratory has shown primary cilia to be extremely rare or totally absent in select cell populations within breast carcinomas both in vitro and in vivo when compared to normal breast epithelial cell lines and tissues. They and others have further shown key cilia-associated genes, including Gli1, to be mutated in breast cancer along with alterations in function and or expression of critical proteins such as cep290, which regulate the cell cycle and the ability of cells to assemble primary cilia.

The authors report too that a number of studies provide evidence that the absence of primary cilia in cancer cells is not a direct result of proliferative activity in this tumor cell population. More promising is the line of evidence suggesting decreased expression or activation of proteins important for primary cilia assembly may be linked to a decrease in primary cilia formation in cancer cells. In regards to breast cancer the authors summarize what is known regarding alternations in hedgehog signaling and primary cilia's dual role on influencing activation or suppression of tumorigenesis. They hypothesize that Gli1 may contribute to mammary gland neoplastic progression in those tumor cells that lack primary cilia.

The authors also comment on primary cilia and mechanotransduction, canonical and PCP Wnt signaling and migration of smooth muscle cells. Finally, the authors comment on their recent discovery that primary cilia are more frequently found on breast myoepithelial cells when compared to luminal epithelial cells. Their hypothesis is that these cells may have a mechanosensor function and influence crosstalk among the cell populations and the extracellular matrix.

The last manuscript by Evans and Hardy very thoroughly summarize our current understanding of the relationship between dietary fat and more specifically dietary long chain fatty acids and breast cancer neoplastic progression in humans. A synopsis of their ground-breaking work in how stearate modulates these events is alluded to but the bulk of the treatise focuses on the epidemiology of the process and what has been learned from meta-analyses of dietary fat research including multi-center cohort and interventional studies. This part ends with a discussion of the accuracy of such dietary studies.

The second half of the treatise explores dietary fatty acids and their effect on cancer cells in vitro and in rodent models and how they correlate with human disease. The authors also spend considerable time on the end of the cancer progression cascade, i.e. 
metastasis studies, since most early workers focused on initiation and promotion of neoplasia rather than metastasis, the cause of virtually all cancer-related deaths in humans. They make a compelling case for the notion that "...not all dietary saturated fatty acids have negative effects on breast cancer while not all unsaturated fatty acids have positive effects..." This is sobering advise for future investigations and reminds us all of the need for balancing dietary fatty acid to control the risk of breast cancer.

We personally learned a lot which will certainly influence our own work and trust readers, be they experts or novices, will be similarly educated.

\section{Gene P. Siegal}

(Co-Guest Editor)

Department of Pathology

University of Alabama at Birmingham

619 South 19th Street

KB 506, Birmingham

AL 35233

USA

E-mail: gsiegal@uab.edu

\author{
Andra R. Frost \\ (Co-Guest Editor) \\ Department of Pathology \\ University of Alabama at Birmingham \\ 619 South 19th Street \\ KB 506, Birmingham \\ AL 35233 \\ USA \\ E-mail: afrost@uab.edu
}

(C) Siegel and Frost; Licensee Bentham Open.

This is an open access article licensed under the terms of the Creative Commons Attribution Non-Commercial License (http:/creativecommons.org/licenses/by-nc/ 3.0/) which permits unrestricted, non-commercial use, distribution and reproduction in any medium, provided the work is properly cited. 